and University of New South Wales, Sydney, Australia;

${ }^{10}$ Australian Research Council Centre of Excellence in Cognition and its Disorders, Sydney, Australia; ${ }^{11}$ The University of Melbourne, Parkville, Australia; ${ }^{12}$ Macquarie University, North Ryde, Australia; ${ }^{13}$ Florey Institute of Neuroscience and Mental Health, Melbourne, Australia. Contact e-mail: vincent.dore@ csiro.au

Background: Visual assessment is the regulatory authorities' approved method for the interpretation of A $\beta$ PET scans. A tracer-specific visual assessment has to be performed by trained readers. However, accurate visual interpretation of PET scans can be challenging, particularly for less experienced readers. In this study, we investigated the added value of using semiquantitative reports, generated using the image analysis software CapAIBL $\AA$, alongside visual $\mathrm{A} \beta$ image inspection. Methods: Forty-eight subjects with a wide spectrum of dementia diagnoses confirmed by autopsy, were identified from two independent databases. Subjects underwent either a ${ }^{11} \mathrm{C}$-PiB or ${ }^{18} \mathrm{~F}$-florbetaben PET scan; Centiloid (CL) values were generated using CapAIBL. Four readers (CR, PR, TC and VD), blinded to clinicopathological diagnosis, visually categorized all scans in regards to $\mathrm{A} \beta$ plaque load ( 0 no $\mathrm{A} \beta$ load, 1 minor $\mathrm{A} \beta$ load, 2 significant $\mathrm{A} \beta$ load, 3 severe $\mathrm{A} \beta$ load). Grade 0 and 1 were classified as low $\mathrm{A} \beta(\mathrm{A} \beta$-), while grade 2 and 3 as high $\mathrm{A} \beta(\mathrm{A} \beta+)$. Non-expert readers (PR TC and $\mathrm{VD}$ ) did not follow tracer-specific training and were asked to rate confidence of their image interpretation. They were subsequently instructed to reexamine the scans alongside the CapAIBL reports, which included the $C L$ values and surface projection of the $\mathrm{A} \beta$ load $\mathrm{z}$-scores. Results: Correlation of expert visual read with CapAIBL CL yielded optimal threshold values of $25.5 \mathrm{CL}$ between $\mathrm{A} \beta$ - and $\mathrm{A} \beta+$ scans (sensitivity:1.00, specificity:0.95, accuracy:0.98, AUC:0.99) and 45CL between significant and severe $\mathrm{A} \beta$ load (sensitivity:1, specificity:1, accuracy:1, AUC:1). When compared to the expert, the performance of the non-expert group was, sensitivity:0.84, specificity: 0.98 acccuracy:90 and AUC: 0.91 . On the second read, when provided with the CapAIBL reports, these statistics reached $0.96,0.97,0.97$ and 0.97 , respectively. On the first read, the intra-reader reliability (IRR) was (Fleiss's Kappa) 0.64 on the A $\beta$ status and 0.41 on the $\mathrm{A} \beta$ load grade. The IRR reached 0.87 and 0.56 on the last read with CapAIBL reports. All non-expert readers were significantly more confident in their scan interpretation when using CapAIBL report $(\mathrm{p}<0.01)$. Conclusions: The addition of $\mathrm{Ca}-$ pAIBL reports alongside to visual $A \beta$ image inspection improved scan interpretation as well as inter-reader reliability among nonexpert readers.

\section{IC-P-009 COMPARING THE CENTILOID SCALE FOR PITTSBURGH COMPOUND B AND FLORBETAPIR IN LONGITUDINAL PET STUDIES OF SPORADIC AD}

Shaney Flores ${ }^{1}$, Yi Su ${ }^{1}$, Guoqiao Wang ${ }^{2}$, Russ C. Hornbeck ${ }^{3}$, Ben Speidel ${ }^{4}$, Andrei G. Vlassenko ${ }^{5}$, Brian A. Gordon ${ }^{1}$, Mark A. Mintun ${ }^{6}$,

Robert A. Koeppe ${ }^{7}$, William E. Klunk ${ }^{8}$, Chengjie Xiong ${ }^{9}$, John C. Morris ${ }^{10}$, Randall J. Bateman ${ }^{11}$, Tammie L. S. Benzinger ${ }^{1},{ }^{1}$ Washington University in St. Louis School of Medicine, St. Louis, MO, USA; ${ }^{2}$ Washington University in St. Louis, St. Louis, MO, USA; ${ }^{3}$ Washington University School of Medicine, St. Louis, MO, USA ${ }^{4}$ Washington University School of Medicine, Saint Louis, MO, USA; ${ }^{5}$ Hope Center for Neurological Disorders, St. Louis, MO, USA; ${ }^{6}$ Avid Radiopharmaceuticals, Philadelphia, PA, USA; ${ }^{7}$ University of Michigan, Ann Arbor, MI, USA; ${ }^{8}$ University of Pittsburgh School of Medicine, Pittsburgh, PA, USA; ${ }^{9}$ Department of Biostatistics, Washington University, St. Louis, MO, USA; ${ }^{10}$ Knight Alzheimer's Disease
Research Center, Saint Louis, MO, USA $;{ }^{11}$ Washington University, St. Louis, MO,USA. Contact e-mail: sflores@wustl.edu

Background: Amyloid imaging with Positron Emission Tomography (PET) plays a critical role in research and clinical assessment of Alzheimer's disease (AD). Radiopharmaceutical tracers, such as $\left[{ }^{11} \mathrm{C}\right]$ Pittsburgh Compound $\mathrm{B}(\mathrm{PiB})$ and $\left[{ }^{18} \mathrm{~F}\right]$ florbetapir, were developed to provide in vivo quantitative estimates of amyloid in humans as an AD biomarker. However, differences in tracer properties and analysis methods produce significant variability in estimates of amyloid burden. To address this issue, the Centiloid Working Group recommended amyloid SUVRs be converted to a standardized scale called Centiloid ${ }^{1}$ for across tracer comparisons. We provide the first comparison of Centiloid values, without and with partial volume correction (PVC), for PiB and florbetapir in longitudinal PET studies of sporadic AD. Methods: Baseline and follow-up longitudinal florbetapir $(\mathrm{N}=26)$ and $\mathrm{PiB}(\mathrm{N}=54)$ were obtained from a sporadic $\mathrm{AD}$ cohort with high amyloid burden at baseline. SUVRs were calculated between 50-70 minutes post-injection for florbetapir and 30-60 minutes post-injection for $\mathrm{PiB}$ with cerebellar grey as a reference and a regional spread function applied for PVC. A 3D sagittal T1-weighted head MR was also acquired to derive regions to average into a global cortical measure of amyloid. The average cortical non-PVC and PVC SUVRs were converted to Centiloid for $\mathrm{PiB}$ and florbetapir. Annualized rate of change was derived from change in PVC Centiloid over time for each participant from a simple linear model and assessed by paired t-tests. Results: Application of PVC reduced overall intra-individual variability and increased the effect sizes of rates of change for both $\mathrm{PiB}$ and florbetapir. However, the rates of change were statistically significant only in $\mathrm{PiB}(\mathrm{p}<.0001$ for both $\mathrm{PVC}$ and non-PVC, respectively). Rate of change was not significantly different between the two tracers $(\mathrm{p}=0.55)$ for PVC Centiloid but greater variability existed within the florbetapir measurement. Conclusions: Florbetapir amyloid measurements in general had higher variability that significantly affected the tracer's ability to detect subtle amyloid burden compared to PiB. Further studies are needed to characterize tracer performance longitudinally. References: ${ }^{1}$ Klunk WE, Koeppe RA, Price JC, Benzinger TLS, Devous Sr MD, Jagust WJ, et al. The Centiloid Project: Standardizing quantitative amyloid plaque estimation by PET. Alzheimer Dement 2015;11:P1-15.
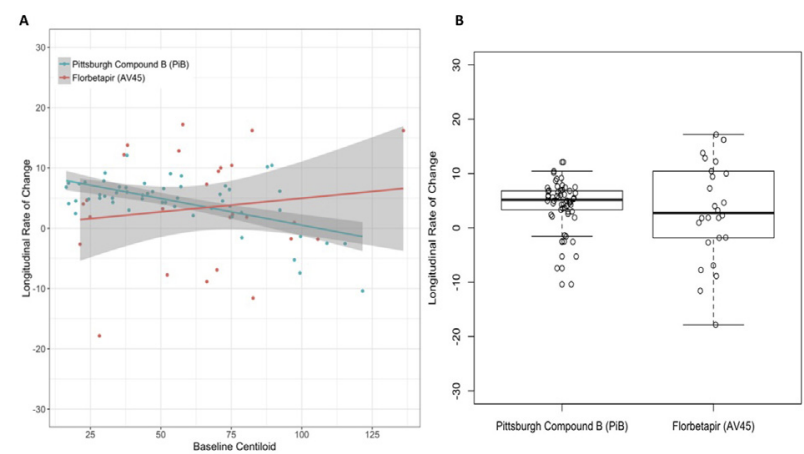

Figure 1. Annualized rate of change of amyloid burden using PiB and florbetapir in the longitudinal sporadic AD cohort. A) As a function of baseline amyloid in Centiloid. Florbetapir showed greater longitudinal variability than PiB. B) Box plot of annualized rate of change measurement for the two tracers. Despite greater variability within the annualized rate of change for florbetapir, both $\mathrm{PiB}$ and florbetapir showed comparable annualized rate of change on the Centiloid scale $(\mathrm{p}=0.55)$. 